\title{
Quantum mechanics with uniform forces
}

\author{
Mark Andrews ${ }^{\text {a) }}$ \\ Department of Quantum Science, Australian National University, Canberra, \\ Australian Capital Territory 0200, Australia
}

(Received 7 February 2010; accepted 2 August 2010)

\begin{abstract}
For a nonrelativistic particle acted on by a spatially uniform but possibly time-dependent force, or for a harmonic oscillator also subject to such a uniform force, the wave function differs from that without the force only by a spatial displacement and a phase factor. The shifts in position and phase are simply derived from a solution of the classical equations of motion. (C) 2010 American Association of Physics Teachers.
\end{abstract}

[DOI: $10.1119 / 1.3481703]$

\section{INTRODUCTION}

The two systems considered here are a nonrelativistic particle acted on by a force that is uniform in space but varies in time and a harmonic oscillator subject to a uniform but timedependent force, often referred to as the forced harmonic oscillator. We show that if the uniform force is turned on at a certain time, then the wave function with the force can easily be obtained from the wave function without the force.

Gregório and de Castro ${ }^{1}$ derived the evolution operator for a particle with a time-varying uniform force. Recently, Bowman ${ }^{2}$ showed that the wave function changes only by a spatial shift and a change in the phase. The forced harmonic oscillator is often treated in textbooks, but generally these treatments do not show the simple effect on the wave function. Carruthers and Nieto ${ }^{3}$ studied the forced oscillator and its relation to coherent states. ${ }^{4}$ In this paper, we give a simpler approach that treats both systems in the same way.

We proceed by making the hypothesis that a uniform force $f(t)$ affects the wave function only by a shift $\bar{x}(t)$ in position and a phase shift $\theta(x, t)$. We then use Ehrenfest's theorem that the expectation values of position and momentum follow a classical trajectory to show that $\bar{x}(t)$ must be the classical shift in position due to $f(t)$ and relate $\theta(x, t)$ to the classical shift in momentum due to $f(t)$. The hypothesis is verified by inserting the trial form of the wave function into Schrödinger's equation.

Although the dependence of the phase $\theta(x, t)$ on $x$ is determined by Ehrenfest's theorem, there remains an added term $\beta(t)$. This term can be determined by substitution into Schrödinger's equation, but an alternative approach to finding the effect of uniform forces via the propagator shows that $\beta(t)$ can be simply expressed in terms of the classical Lagrangian of the system. This approach, which requires more formalism, is discussed in the Appendix.

\section{APPLYING EHRENFEST'S THEOREM}

Our hypothesis is that the wave function has the form $\psi(x, t)=\exp [i \theta(x, t)] \Psi(x-\bar{x}, t)$, where $\Psi(x, t)$ is the wave function without the uniform force $f(t)$. Thus, $\bar{x}(t)$ is the shift in position caused by $f(t)$, and $\theta(x, t)$ is real and is the phase shift caused by $f(t)$. Changing the variable in $\int_{-\infty}^{\infty}|\psi(x, t)|^{2} x d x$ from $x$ to $x-\bar{x}$ leads to $\langle\hat{x}\rangle_{\psi}=\langle\hat{x}\rangle_{\Psi}+\bar{x}$. (The symbol $\hat{x}$ denotes the operator for the $x$-component of position, and we will generally place a hat on each operator.) Ehrenfest's theorem shows that $\langle\hat{x}\rangle_{\psi}$ follows a classical trajectory with the force $f(t)$, while $\langle\hat{x}\rangle_{\Psi}$ follows a classical trajectory with no uniform force. Thus, $\bar{x}$ is the difference between these two trajectories.

Similarly, $\langle\hat{p}\rangle_{\psi}=\hbar \partial_{x} \theta+\langle\hat{p}\rangle_{\Psi}$ and the change in $\langle\hat{p}\rangle$ for the wave functions with and without the uniform force is $\bar{p}(t)$ $=\hbar \partial_{x} \theta$, and therefore $\hbar \theta=\bar{p} x-\beta(t)$. The unknown $\beta(t)$ will be determined when the trial wave function

$$
\psi(x, t)=\exp \left[\frac{i}{\hbar}(\bar{p}(t) x-\beta(t))\right] \Psi(x-\bar{x}, t)
$$

is substituted into Schrödinger's equation. Ehrenfest's theorem implies that $\bar{p}(t)$ is the difference in momentum between classical trajectories with and without the force. If the force is turned on at $t=0$, then $\bar{x}(t), \bar{p}(t)$ is the classical trajectory such that $\bar{x}(0)=0, \bar{p}(0)=0$.

\section{PARTICLE WITH UNIFORM FORCE}

The classical equations of motion are $m \dot{x}=p$ and $\dot{p}=f(t)$, where $f(t)$ is the force. The classical trajectory is $p=m v_{0}$ $+F(t), x=x_{0}+v_{0} t+F_{1}(t) / m$, where

$$
F(t)=\int_{0}^{t} f\left(t^{\prime}\right) d t^{\prime}, \quad F_{1}(t)=\int_{0}^{t} F\left(t^{\prime}\right) d t^{\prime} .
$$

The change in the momentum due to the force is $\bar{p}(t)=F(t)$ and the displacement is $\bar{x}(t)=F_{1}(t) / m$. If we insert the wave function in Eq. (1) into Schrödinger's equation

$$
i \hbar \frac{\partial \psi}{\partial t}=-\frac{\hbar^{2}}{2 m} \frac{\partial^{2} \psi}{\partial x^{2}}-f(t) x \psi
$$

and regard $\Psi$ as a function of $\xi=x-\bar{x}$ and $t$, we obtain

$$
i \hbar \frac{\partial \Psi}{\partial t}=-\frac{\hbar^{2}}{2 m} \frac{\partial^{2} \Psi}{\partial \xi^{2}}+\left(\bar{p}^{2} / 2 m-d_{t} \beta\right) \Psi,
$$

where we have used the classical equations $m \dot{\bar{x}}=\bar{p}$ and $\dot{\bar{p}}$ $=f(t)$. Equation (4) becomes the free-particle evolution equation if $\beta(t)=F_{2}(t) / 2 m$, where

$$
F_{2}(t)=\int_{0}^{t} F\left(t^{\prime}\right)^{2} d t^{\prime} .
$$

Thus, the wave function with the uniform force $f(t)$ has the form given in Eq. (1), where $\Psi(x, t)$ is the free-particle wave function, and 


$$
\bar{p}=F(t), \quad \bar{x}=F_{1}(t) / m, \quad \beta=F_{2}(t) / 2 m .
$$

Note that $\psi(x, 0)=\Psi(x, 0)$, as required.

Example 1. A constant uniform force. If a uniform force is turned on at time $t=0$ and then remains at the constant value $f$, the wave function for $t>0$ is

$$
\psi(x, t)=\exp \left[\frac{i}{\hbar}\left(f t x-\frac{f^{2} t^{3}}{6 m}\right)\right] \Psi\left(x-\frac{f t^{2}}{2 m}, t\right),
$$

where $\Psi(x, t)$ is the wave function without the force.

Example 2. An impulsive uniform force. If a free particle with wave function $\Psi(x, t)$ is subject to a uniform force $\lambda \delta\left(t-t^{\prime}\right)$ at time $t=t^{\prime}$, the wave function for $t>t^{\prime}$ becomes

$$
\psi(x, t)=\exp \left[\frac{i}{\hbar}\left(\lambda x-\frac{\lambda^{2}}{2 m}\left(t-t^{\prime}\right)\right)\right] \Psi\left(x-\frac{\lambda}{m}\left(t-t^{\prime}\right), t\right) .
$$

Problem 1. If the force has the constant value $f$ for $0<t$ $<\tau$ and is otherwise zero, calculate the values of the phase shift $\beta$ and the displacement $\int_{0}^{t} F\left(t^{\prime}\right) d t^{\prime} / m$ for $t>0$. Take $f$ $=\lambda / \tau$, so that $\int_{0}^{t} f\left(t^{\prime}\right) d t^{\prime}=\lambda$ for $t>\tau$, and show that as $\tau$ $\rightarrow 0$, the phase shift and displacement agree with the result in Example 2.

\section{THE MOMENTUM WAVE FUNCTION}

Corresponding to any spatial wave function $\psi(x, t)$, the momentum wave function $\phi(p, t)$ is

$$
\phi(p, t)=\frac{1}{\sqrt{2 \pi \hbar}} \int_{-\infty}^{\infty} \exp \left(-\frac{i}{\hbar} p x\right) \psi(x, t) d x .
$$

We substitute $\psi$ into Eq. (1), rearrange the exponent, and obtain

$$
\begin{aligned}
\phi(p, t) & =e^{i \gamma(p, t)} \frac{1}{\sqrt{2 \pi \hbar}} \int_{-\infty}^{\infty} \exp \left[-\frac{i}{\hbar}(p-\bar{p}) \xi\right] \Psi(\xi, t) d x \\
& =e^{i \gamma(p, t)} \Phi(p-\bar{p}(t), t)
\end{aligned}
$$

where $\Phi(p, t)$ is the momentum wave function without the force, and

$$
\hbar \gamma(p, t)=-[p-\bar{p}(t)] \bar{x}(t)-\beta(t) .
$$

Thus, the effect of a uniform time-dependent force on the momentum wave function is only a displacement in momentum and a phase change.

Problem 2. The momentum wave function with a uniform force can be treated by a method similar to that for the spatial wave function. The force causes the momentum to increase by $F(t)$, and hence we introduce the variable $\rho=p-F(t)$. (a) If the effect of $f(t)$ is a phase shift, show that the shift must have the form $-[\bar{x} p-\gamma(t)] / \hbar$. (b) Show that the function $\phi(p, t)=\exp [-i(\bar{x} p-\gamma) / \hbar] \Phi(\rho, t)$ satisfies the evolution equation provided that $\Phi(\rho, t)$ satisfies the free-particle equation $i \hbar \partial_{t} \Phi=\left(\rho^{2} / 2 m\right) \Phi$ and $d_{t} \gamma(t)=\bar{x} f(t)+F(t)^{2}$. (c) Show that this $\gamma(p, t)$ agrees with Eq. (11).

\section{OSCILLATOR AND UNIFORM FORCE}

A similar approach can be used with the Hamiltonian,

$$
\hat{H}=\frac{\hat{p}^{2}}{2 m}+\frac{1}{2} m \omega^{2} \hat{x}^{2}-f(t) \hat{x}
$$

for the forced harmonic oscillator. The shifts $\bar{x}$ and $\bar{p}$ are the solution with zero initial position and momentum of the classical equation of motion $m \dot{x}=p$ and $\dot{p}=-m \omega^{2} x+f(t)$. Thus, $\bar{x}=S(t) / m \omega$ and $\bar{p}=C(t)$, where

$$
\begin{aligned}
& S(t)=\int_{0}^{t} f\left(t^{\prime}\right) \sin \omega\left(t-t^{\prime}\right) d t^{\prime}, \\
& C(t)=\int_{0}^{t} f\left(t^{\prime}\right) \cos \omega\left(t-t^{\prime}\right) d t^{\prime} .
\end{aligned}
$$

We insert $\psi(x, t)$ in Eq. (1) into Schrödinger's equation,

$$
i \hbar \frac{\partial \psi}{\partial t}=-\frac{\hbar^{2}}{2 m} \frac{\partial^{2} \psi}{\partial x^{2}}+\frac{1}{2} m \omega^{2} x^{2} \psi-f(t) x \psi
$$

and treat $\Psi$ as a function of $\xi=x-\bar{x}$ and $t$. The result is that $\Psi(\xi, t)$ satisfies Schrödinger's equation for the oscillator without the uniform force provided that

$$
\frac{d \beta}{d t}=\frac{1}{2 m} \bar{p}^{2}-\frac{1}{2} m \omega^{2} \bar{x}^{2} .
$$

The equations of motion give $d_{t}(\bar{p} \bar{x})=\bar{p}^{2} / m-m \omega^{2} \bar{x}^{2}+f(t) \bar{x}$, and therefore we have the alternative form

$$
\beta(t)=\frac{1}{2} \bar{p} \bar{x}-\frac{1}{2} \int_{0}^{t} f\left(t^{\prime}\right) \bar{x}\left(t^{\prime}\right) d t^{\prime} .
$$

Example 3. If a harmonic oscillator with wave function $\Psi(x, t)$ is subject to an impulsive uniform force $\lambda \delta(t)$ at time $t=0$, the wave function will become, for $t>0, \psi(x, t)$ $=\exp [i \theta(x, t)] \Psi(x-(\lambda / m \omega) \sin \omega t, t), \quad$ where $\hbar \theta(x, t)$ $=\lambda x \cos \omega t-\sin 2 \omega t / 4 m \omega$.

Problem 3. Show that the effect of a time-dependent uniform force on the momentum wave function of a harmonic oscillator is just a shift of $\bar{p}$ in momentum and a change $\frac{1}{2} \bar{p} \bar{x}+\frac{1}{2} \int_{0}^{t} f\left(t^{\prime}\right) \bar{x}\left(t^{\prime}\right) d t^{\prime}$ in the phase, where $\bar{p}=C(t)$ and $\bar{x}$ $=S(t) / m \omega$, as in Eqs. (13) and (14).

Exercise 4. Show that $\left\langle(\hat{x}-\langle\hat{x}\rangle)^{2}\right\rangle$ and $\left\langle(\hat{p}-\langle\hat{p}\rangle)^{2}\right\rangle$ are not affected by any uniform force, either for the oscillator or the free particle.

\section{COHERENT STATES}

If a harmonic oscillator, initially in its ground state with wave function $\Psi_{0}(x, 0)=\exp \left(-\frac{1}{2} x^{2} / \sigma^{2}\right)$, where $\sigma^{2}=\hbar / m \omega$, is subject to a uniform force, then $|\psi(x, t)|$ and $|\phi(p, t)|$ remain Gaussian with unchanged scale but displaced by $\bar{x}(t)$ and $\bar{p}(t)$, respectively. These states, obtained by applying any uniform force to the oscillator's ground state, are called coherent states. The result of applying a uniform force to any coherent state will also produce a coherent state because this uniform force can be taken as a continuation of the force that produced the initial coherent state. 
From Eq. (1), the wave function with the force applied is $\quad \psi(x, t)=\exp [i(\bar{p} x-\beta) / \hbar] \Psi_{0}(x-\bar{x}, t), \quad$ where $\quad \Psi_{0}(x, t)$ $=\exp \left(-\frac{1}{2} i \omega t-\frac{1}{2} x^{2} / \sigma^{2}\right]$. Then $\psi(x, t)=\hat{A} \Psi_{0}(x, t)$, where

$$
\hat{A}=\exp \left[-\frac{i}{\hbar} \beta-\frac{1}{2} \frac{\bar{x}^{2}}{\sigma^{2}}+\left(\frac{\bar{x}}{\sigma}+\frac{i}{\hbar} \sigma \bar{p}\right) \frac{\hat{x}}{\sigma}\right] .
$$

We see that a coherent state is an eigenstate, with eigenvalue $\bar{a}=(\bar{x} / \sigma+i \sigma \bar{p} / \hbar) / \sqrt{2}$, of the oscillator lowering operator $\hat{a}$ $=(\hat{x} / \sigma+i \sigma \hat{p} / \hbar) / \sqrt{2}$, because $[\hat{a}, \hat{A}]=\bar{a} \hat{A}$ and $\hat{a} \Psi_{0}=0$, also $\left[\hat{a}, \hat{a}^{\dagger}\right]=1$. The eigenvalue for the result of applying two successive forces is the sum $\bar{a}_{1}+\bar{a}_{2}$ of the eigenvalues $\bar{a}_{1}$ and $\bar{a}_{2}$ for each force because the classical shifts in position and momentum add. If we apply a force that shifts the position by $\bar{x}_{1}$ and the momentum by $\bar{p}_{1}$ and then apply a force that shifts by $\bar{x}_{2}$ and $\bar{p}_{2}$, the total shift is $\bar{x}_{1}+\bar{x}_{2}$ and $\bar{p}_{1}+\bar{p}_{2}$.

This analysis of the coherent states has used the explicit form of the ground state wave function, but the algebra of the oscillator applies also in the quantum theory of electromagnetic radiation, where wave functions are not commonly used and the operators $\hat{a}$ and $\hat{a}^{\dagger}$ are preferred to $\hat{x}$ and $\hat{p}$. To connect with such an analysis, we first recast Eq. (1) as $\psi(x, t)=\hat{A} \Psi(x, t)$, where

$$
\hat{A}=e^{-i \beta / \hbar} \exp (i \bar{p} \hat{x} / \hbar) \exp (-i \bar{x} \hat{p} / \hbar)
$$

because $\hat{p}$ is the generator of translations. Then we have

$$
\hat{A}=\exp [-i(\bar{p} \bar{x}-\beta /) \hbar] \exp [i(\bar{p} \hat{x}-\bar{x} \hat{p}) / \hbar]
$$

because $[\hat{p}, \hat{x}]=-i \hbar$. Also, $i(\bar{p} \hat{x}-\bar{x} \hat{p}) / \hbar=\bar{a} \hat{a}^{\dagger}-\bar{a}^{\star} \hat{a}$. Now

$$
\hat{D}(\bar{a})=\exp \left(\bar{a} \hat{a}^{\dagger}-\bar{a}^{\star} \hat{a}\right)
$$

is commonly referred to as the displacement operator and is often taken as the starting point for a treatment of coherent states (suitable for the oscillator and for photons). One can show that $[\hat{a}, \hat{D}(\bar{a})]=\bar{a} \hat{D}(\bar{a})$, and hence $\hat{D}(\bar{a}) \Psi_{0}$ is an eigenstate of $\hat{a}$ if $\Psi_{0}$ is the ground state of the oscillator. Similarly, $\hat{D}\left(\bar{a}_{1}\right) \hat{D}\left(\bar{a}_{2}\right)$ equals $\hat{D}\left(\bar{a}_{1}+\bar{a}_{2}\right)$, apart from a phase factor, so that the eigenvalues of successive displacements add, in agreement with our previous result. These and other calculations, such as expanding the coherent states in terms of energy eigenstates, can be found in many textbooks. ${ }^{4}$

\section{DISCUSSION}

The result in Sec. III on the change in the wave function of a free particle can easily be derived from the expression derived by Gregório and de Castro ${ }^{1}$ for the evolution operator. Their Eq. (8) in our notation is

$$
|\psi(t)\rangle=\exp \left[\frac{i}{\hbar}(\bar{p} x-\beta)\right] \exp \left[-\frac{i}{\hbar}\left(\hat{H}_{0} t+\bar{x} \hat{p}\right)\right]|\psi(0)\rangle,
$$

where $\hat{H}_{0}=\hat{p}^{2} / 2 m$. But $\hat{H}_{0}$ commutes with $\hat{p}$, and thus $\exp [$ $\left.-i\left(\hat{H}_{0} t+\bar{x} \hat{p}\right) / \hbar\right]=\exp \left[-i \hat{H}_{0} t / \hbar\right] \exp [-i \bar{x} \hat{p} / \hbar]$, while in the Schrödinger representation $\exp [-i \bar{x} \hat{p} / \hbar] \psi(x, t)=\psi(x-\bar{x}, t)$. Because $\exp \left[-i \hat{H}_{0} t / \hbar\right]$ is the free evolution operator, we obtain Eq. (1) with the correct $\beta(t)$.
The result in Problem 4 that the two second-order moments are not affected by uniform forces was also given (for the case of a free particle) in Ref. 1. In addition to the second-order moments, all moments about the centroid are unaffected by a uniform force. For $\left\langle(\hat{x}-\langle\hat{x}\rangle)^{n}\right\rangle$, this result follows from the form of the wave function in Eq. (1): $\left\langle\psi\left|(x-\langle x\rangle)^{n}\right| \psi\right\rangle=\left\langle\Psi\left|(\xi-\langle\xi\rangle)^{n}\right| \Psi\right\rangle$. The same result applies for $\left\langle(\hat{p}-\langle\hat{p}\rangle)^{n}\right\rangle$ using the form of the momentum wave function. Moments of the form $\left\langle(\hat{x}-\langle\hat{x}\rangle)^{n}(\hat{p}-\langle\hat{p}\rangle)^{k}\right\rangle$ are not so simple, but first verifying that $\left(\hat{p}_{x}-\langle\hat{p}\rangle_{\psi}\right)^{k} \psi=\exp [i(\bar{p} x-\beta) / \hbar]\left(\hat{p}_{\xi}\right.$ $\left.-\langle\hat{p}\rangle_{\Psi}\right)^{k} \Psi$ makes the calculation straightforward. That all moments about the centroid are unaffected is implied by the fact that Heisenberg's operator equations of motion for the position and momentum relative to the centroid are independent of any uniform forces, as in the classical case.

Our results can easily be extended to more dimensions and any isotropic system with a Hamiltonian that is at most quadratic in position and momentum (with arbitrary timedependence in the coefficients),

$$
\hat{H}=\frac{1}{2} a \hat{\mathbf{p}}^{2}+\frac{1}{2} b(\hat{\mathbf{p}} \cdot \hat{\mathbf{x}}+\hat{\mathbf{x}} \cdot \hat{\mathbf{p}})+\frac{1}{2} c \hat{\mathbf{x}}^{2}+\mathbf{f} \cdot \hat{\mathbf{p}}+\mathbf{g} \cdot \hat{\mathbf{x}} .
$$

If $\Psi(\mathbf{x}, t)$ satisfies Schrödinger's equation with no linear terms, then $\psi(\mathbf{x}, t)=\exp [i(\overline{\mathbf{p}} \cdot \mathbf{x}-\beta(t)) / \hbar] \Psi(\mathbf{x}-\overline{\mathbf{x}}, t)$ will satisfy Schrödinger's equation with the linear terms included, for times $t \geq 0$, where $\overline{\mathbf{x}}(t), \overline{\mathbf{p}}(t)$ is the solution with $\overline{\mathbf{x}}\left(t_{0}\right)$ $=\mathbf{0}, \overline{\mathbf{p}}\left(t_{0}\right)=\mathbf{0}$ of the classical equations of motion $d_{t} \overline{\mathbf{x}}=a \overline{\mathbf{p}}$ $+b \overline{\mathbf{x}}+\mathbf{f}$ and $-d_{t} \overline{\mathbf{p}}=b \overline{\mathbf{p}}+c \overline{\mathbf{x}}+\mathbf{g}$, while $\dot{\beta}=\frac{1}{2} a \hat{\mathbf{p}}^{2}-\frac{1}{2} c \hat{\mathbf{x}}^{2}-\mathbf{g} \cdot \hat{\mathbf{x}}$. This expession for $\dot{\beta}$ is the Lagrangian of the corresponding classical system, and hence

$$
\beta(t)=\int_{t_{0}}^{t} L\left(\overline{\mathbf{x}}\left(t^{\prime}\right), \dot{\overline{\mathbf{x}}}\left(t^{\prime}\right), t^{\prime}\right) d t^{\prime},
$$

where $L(\mathbf{x}, \dot{\mathbf{x}}, t)$ is the classical Lagrangian. This connection will be explained in the Appendix.

\section{SUMMARY}

For a free particle or a harmonic oscillator, if an additional (possibly time-dependent) uniform force is turned on at time $t=0$, the wave function at later times is

$$
\psi(x, t)=\exp \left[\frac{i}{\hbar}(\bar{p}(t) x-\beta(t))\right] \Psi(x-\bar{x}, t),
$$

where $\Psi(x, t)$ is the wave function without the force and $\bar{x}(t), \bar{p}(t)$ is the classical trajectory with $\bar{x}(0)=0$ and $\bar{p}(0)=0$. Also, $\beta(t)=\int_{0}^{t} L\left(\bar{x}\left(t^{\prime}\right), \dot{\bar{x}}\left(t^{\prime}\right), t^{\prime}\right) d t^{\prime}$, where $L(x, \dot{x}, t)$ is the Lagrangian of the classical system including the uniform force.

\section{APPENDIX: USING THE PROPAGATOR}

The propagator $K\left(x, t: x^{\prime}, t^{\prime}\right)$ is such that

$$
\psi(x, t)=\int_{-\infty}^{\infty} K\left(x, t: x^{\prime}, t^{\prime}\right) \psi\left(x^{\prime}, t^{\prime}\right) d x^{\prime} .
$$

For any Hamiltonian that is at most quadratic in position and momentum, the propagator can be found from the classical Lagrangian $L(x, \dot{x}, t)$, 


$$
K\left(x, t: x^{\prime}, t^{\prime}\right)=\frac{1}{\sqrt{2 \pi i \hbar \xi(t)}} \exp \left[\frac{i}{\hbar} S\left(x, t: x^{\prime}, t^{\prime}\right)\right],
$$

where $\xi(t)=\left|\partial^{2} S / \partial x \partial x^{\prime}\right|^{-1}$ is a function of $t$ only, and

$$
S\left(x, t: x^{\prime}, t^{\prime}\right)=\int_{x^{\prime}, t^{\prime}}^{x, t} L(x(\tau), \dot{x}(\tau), \tau) d \tau .
$$

The integration is taken along the classical trajectory from $x^{\prime}, t^{\prime}$ to $x, t$. Equation (A2) was first proposed by Van Vleck ${ }^{5}$ as a semiclassical approximation to the propagator (not limited to quadratic Hamiltonians) and was shown by Morette to be exact in the quadratic case. It is similar to Feynman's sum over paths. In the semiclassical limit, or exactly for quadratic Hamiltonians, only the classical path contributes to the path integral.

We now show that Eq. (A2) for the propagator leads to the result in Eq. (1). Because we have been working with the momentum $p$ rather than the velocity $v$, we will use $\mathcal{L}(x, p, t)=L(x, \dot{x}, t)$. For the forced harmonic oscillator, the Lagrangian is

$$
\mathcal{L}(x, p, t)=\frac{1}{2 m} p^{2}-\frac{1}{2} m \omega^{2} x^{2}+f(t) x .
$$

In terms of $X=x-\bar{x}$ and $P=p-\bar{p}$,

$$
\mathcal{L}(x, p, t)=\mathcal{L}_{0}(X, P, t)+\frac{d}{d t}(\bar{p} x)-\mathcal{L}_{0}(\bar{x}, \bar{p}, t),
$$

where $\mathcal{L}_{0}(X, P, t)=P^{2} / 2 m-\frac{1}{2} m \omega^{2} X^{2}$ is the Lagrangian without the driving force $f(t)$. Because the integration over $x^{\prime}$ in Eq. (A1) involves only the first two terms in Eq. (A5), the result in Eq. (1) follows with $\dot{\beta}=\mathcal{L}_{0}(\bar{x}, \bar{p}, t)$, in agreement with Eq. (16). This analysis also applies when the oscillator force is absent (equivalent to taking $\omega=0$ ) and can be extended to any quadratic Hamiltonian, even with arbitrary time-dependent coefficients.

\footnotetext{
a) Electronic mail: mark.andrews@anu.edu.au

${ }^{1}$ M. A. Gregório and A. S. de Castro, "A particle moving in a homogeneous time-varying force,” Am. J. Phys. 52, 557-559 (1984).

${ }^{2}$ Gary E. Bowman, "Quantum-mechanical time evolution and uniform forces,” J. Phys. A 39, 157-162 (2006).

${ }^{3}$ P. Carruthers and M. M. Nieto, "Coherent states and the forced quantum oscillator,” Am. J. Phys. 33, 537-544 (1965).

${ }^{4}$ Eugen Merzbacher, Quantum Mechanics, 3rd ed. (Wiley, New York, 1998), Sec. 10.7.

${ }^{5}$ J. H. Van Vleck, "The correspondence principle in the statistical interpretation of quantum mechanics," Proc. Natl. Acad. Sci. U.S.A. 14, 178188 (1928).

${ }^{6} \mathrm{C}$. Morette, "On the definition and approximation of Feynman's path integrals," Phys. Rev. 81, 848-852 (1951).
}

\section{MAKE YOUR ONLINE MANUSCRIPTS COME ALIVE}

A picture is worth a thousand words. Film or animation can be worth much more. If you submit a manuscript which includes an experiment or computer simulation, why not make a film clip of the experiment or an animation of the simulation, and place it on the Supplementary Material server. Your online manuscript will have a direct link to your supplementary material.

See http://www.kzoo.edu/ajp/EPAPS.html for more information. 